\title{
Para-aortic Lymphadenectomy for Gynecologic Cancers: Introducing the "Trans-Retro-Peritoneal (TRP) Single-Port Access"
}

\author{
Loïc Lelievre, MD 무, and Patrice Mathevet, MD, PhD \\ Department of Gynaecology, Centre Hospitalier Universitaire Vaudois, Lausanne, Switzerland
}

\begin{abstract}
Background. In gynecological surgery to date, two distinct types of endoscopic accesses have been used to perform para-aortic lymphadenectomies: transperitoneal and extraperitoneal, each with advantages and disadvantages. ${ }^{1}$ We propose to develop a new mini-invasive access to perform an endoscopic extraperitoneal para-aortic lymphadenectomy via a single-port umbilical device that, to our knowledge, has never been described.
\end{abstract}

Methods. This innovative approach combines both an extraperitoneal and intraperitoneal procedure via the same umbilical incision using one single trocar. A $3-4 \mathrm{~cm}$ transumbilical incision is performed and a three-channel singleport device (Gelpoint $\mathrm{Mini}^{\circ}$; Applied Medical, Rancho Santa Margarita, CA, USA) is introduced in the peritoneal cavity. After peritoneal exploration, the peritoneum overlying the aorta bifurcation is grabbed, raised to the umbilicus, opened, and the single-port device is then reintroduced into the retroperitoneal space.

Results. The intervention has been successful in three patients with locally advanced cervical cancer (two Inter-

Electronic supplementary material The online version of this article (https://doi.org/10.1245/s10434-019-07379-8) contains supplementary material, which is available to authorized users.

(C) The Author(s) 2019

First Received: 9 January 2019;

Published Online: 23 April 2019

L. Lelievre, MD

e-mail: loic.lelievre@chuv.ch national Federation of Gynecology and Obstetrics [FIGO] stage IB2, and one FIGO stage IVA) scheduled for concomitant radiochemotherapy after exclusion of any suspicious lymph nodes by $18 \mathrm{~F}$-fluorodeoxyglucose-positron emission tomography (FDG-PET), according to our standards of practice based on the European Society of Gynaecological Oncology (ESGO) and National Comprehensive Cancer Network (NCCN) guidelines. ${ }^{2,3}$ Due to the type of cancer, lymphadenectomy was limited to the inframesenteric nodes, ${ }^{2,4}$ although the dissection went up to the left renal vein in each case. We retrieved 13, 20, and 25 lymph nodes. No complications occurred.

Conclusions. We describe a promising technique that combines all the advantages of the two previously described accesses without their disadvantages, and with the cosmetic benefit of one almost invisible single trans-umbilical scar.

OPEN ACCESS This article is distributed under the terms of the Creative Commons Attribution 4.0 International License (http://crea tivecommons.org/licenses/by/4.0/), which permits unrestricted use, distribution, and reproduction in any medium, provided you give appropriate credit to the original author(s) and the source, provide a link to the Creative Commons license, and indicate if changes were made. The Creative Commons Public Domain Dedication waiver (http://creativecommons.org/publicdomain/zero/1.0/) applies to the data made available in this article, unless otherwise stated.

\section{REFERENCES}

1. Beytout C, Laas E, Naoura I, Bendifallah S, Canlorbe G, Ballester $\mathrm{M}$, et al. Single-port extra- and transperitoneal approach for paraaortic lymphadenectomy in gynecologic cancers: a propensityadjusted analysis. Ann Surg Oncol. 2016;23(3):952-8.

2. Cibula D, Pötter R, Planchamp F, et al. Radiotherapy and Oncology/European Society of Pathology guidelines for the management of patients with cervical cancer. Radiother Oncol. 2018;127:404-16. 
3. Koh WJ, Abu-Rustum NR, Bean S, et al. Cervical cancer, Version 3.2019, NCCN clinical practice guidelines in oncology. $J$ Natl Compr Canc Netw. 2019;17(1):64-84.

4. Azais $\mathrm{H}$, Ghesquiere L, Petitnicolas C, et al. Pretherapeutic staging of locally advanced cervical cancer: inframesenteric paraaortic lymphadenectomy accuracy to detect paraaortic metastases in comparison with infrarenal paraaortic lymphadenectomy. Gynecol Oncol. 2017;147(2):340-4.

Publisher's Note Springer Nature remains neutral with regard to jurisdictional claims in published maps and institutional affiliations. 